\title{
Transition professionnelle et emploi des descendants d'immigrés en France
}

Yaël Brinbaum et Jean-Luc Primon

\section{(2) OpenEdition}

1 Journals

Édition électronique

URL : http://journals.openedition.org/ress/2338

DOI : $10.4000 /$ ress.2338

ISSN : 1663-4446

Éditeur

Librairie Droz

Édition imprimée

Date de publication : 1 juin 2013

Pagination : 33-63

ISBN : 978-2-600-01749-7

ISSN : 0048-8046

\section{Référence électronique}

Yaël Brinbaum et Jean-Luc Primon, «Transition professionnelle et emploi des descendants d'immigrés en France », Revue européenne des sciences sociales [En ligne], 51-1 | 2013, mis en ligne le 01 janvier 2017, consulté le 10 décembre 2020. URL : http://journals.openedition.org/ress/2338 ; DOI https://doi.org/10.4000/ress.2338

\section{(c) Librairie Droz}




\title{
TRANSITION PROFESSIONNELLE ET EMPLOI DES DESCENDANTS D'IMMIGRÉS EN FRANCE
}

\author{
YAËL BRINBAUM \& JEAN-LUC PRIMON \\ Centre d'Études de l'Emploi / INED \\ \& Université de Nice Sophia-Antipolis-URMIS/IRD \\ yael.brinbaum@cee-recherche.fr/primon@unice.fr
}

\begin{abstract}
Résumé. L'article traite de la question de la transition de l'éducation à l'emploi et de la situation sur le marché du travail français des descendants d'immigrés âgés de 18 à 35 ans. Avec pour références le modèle théorique de l'assimilation segmentée et les recherches qui mettent l'accent sur les discriminations à l'embauche en fonction de l'origine migratoire de certaines catégories de population, l'étude revient sur le comportement d'activité, l'accès à l'emploi, le chômage et les formes d'emploi des descendants d'immigrés en exploitant les données d'une grande enquête statistique de population spécialement conçue pour connaître les immigrés et les descendants d'immigrés: l'enquête «Trajectoires et Origines» (TeO, INED/INSEE, 2008). Les résultats confirment la pénalisation socio-économique des descendants des immigrations nordafricaines et subsahariennes et la segmentation des destins socio-professionnels, mais montre aussi le caractère sexué des divisions à l'intérieur des groupes de population.
\end{abstract}

Mots-clés: transition professionnelle, marché du travail, descendants d'immigrés, pénalisation, discrimination, assimilation segmentée.

\begin{abstract}
This paper deals with the question of the transition from school to work and the situation on the labour market of Second-Generation Immigrants youth. Based on the "segmented assimilation theory" and on research that emphasize discrimination according to ethnic origins, the study analyses the access to work, unemployment and conditions of work of the descendants of immigrants. We use the data of the "Trajectories and Origins" Survey (TeO, INSEE/INED, 2008), a big quantitative survey on the immigrants and the descendants of immigrants. Outcomes confirm the socio-economic penalty of the descendants of immigrants from North Africa and Saharan Africa and the segmentation of their socio economic destiny, but show also the gendered division among the groups.
\end{abstract}

Keywords: school-to-work transition, labour market, descendants of immigrants, penalty, discrimination, segmented assimilation. 


\section{INTRODUCTION L'assimilation segmentée et le barrage des discriminations}

La transition de l'éducation à l'emploi et les débuts dans la vie professionnelle des descendants d'immigrés en France ont fait l'objet depuis vingt cinq ans de nombreuses publications basées sur des sources statistiques de plus en plus détaillées. Progressivement, les recherches ont délaissé les problématiques de l'intégration érigées en modèle politique - elles-mêmes compatibles avec la vision classique de l'insertion professionnelle comme processus individuel de stabilisation professionnelle plus ou moins long - pour mettre l'accent sur la pluralité des trajectoires d’accès au marché du travail et aussi sur les barrages à l'emploi qui se manifestent notamment sous la forme de discriminations sélectives qui pénalisent socio-économiquement certains groupes, comme les descendants de nord-africains.

La référence au modèle théorique dit de «l'assimilation segmentée» (Portes, Zhou, I993 p. 74-96; Zhou, 1997, p.975-1008) est devenue de plus en plus fréquente dans les travaux les plus récents qui étudient l'accès à l'emploi des descendants des populations immigrées. Développée par la sociologie économique de l'immigration aux États-Unis au cours des années 1990, cette théorie rompt avec la vision à la fois linéaire et uniforme de l'inclusion des populations issues des immigrations qui représente l'assimilation comme un processus inter-générationnel et temporel conduisant à une indifférenciation multidimensionnelle des différentes composantes dans la société d'immigration. Or, de nombreux résultats d'enquêtes aux États-Unis ont montré que les enfants d'immigrés de certains groupes, manifestement minorisés, en particulier lorsque leur capital scolaire était faible, parvenaient difficilement à accéder à un emploi qui les inscrivait dans un courant de mobilité sociale leur permettant d'occuper une place significativement différente de celle de leurs parents immigrés : en réalité, «l’assimilation» s’apparenterait plus à un processus de segmentation ou de division des populations issues des immigrations (deuxième génération), au cours duquel certains groupes sont favorisés et d'autres désavantagés, 


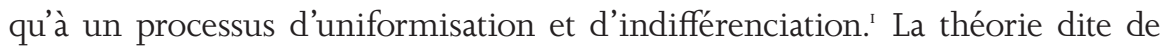
«l'assimilation segmentée » vise à étudier l'accès aux biens sociaux des descendants d'immigrés comme un phénomène structuré historiquement et socialement et non strictement individuel et s'intéresse aux différents «modes d'incorporation» en vigueur dans la société d'immigration. En tant que schéma d’analyse, elle se compose généralement de trois parties: I. une identification des facteurs externes qui conditionnent l'accès aux biens : structure familiale, capital sociaux, scolaires, économiques des familles immigrées, etc.; 2. une identification des barrages sociaux, économiques auxquels se confrontent les enfants d'immigrés: racisme; marché du travail segmenté et précarisé, activités déviantes, criminalisation, etc. ; 3. une prédiction des différentes trajectoires et destinées, compte tenu des différents forces et facteurs en présence (Portes, Fernandez-Kelly, Haller, 2009, p. I077-II04). De cette approche novatrice, nous retiendrons l'accent mis sur les barrières dans la relation à l'emploi et sur les bifurcations des trajectoires pouvant conduire à une infériorisation des positions pour certaines catégories des descendants d'immigrés alors que d'autres s'inscrivent dans un courant de mobilité sociale ascendante.

En France, jusqu'aux années 1990, les sources statistiques permettant d’analyser le passage de l'école à l'emploi des secondes générations étaient très rares en raison des habitudes de la statistique française qui n'enregistrait pas l'origine migratoire des parents. À partir du milieu des années 1990 et un repérage plus systématique des immigrés («personnes nées étrangères à l'étranger») dans la statistique publique, les choses vont changer. En 1992, est réalisée une grande enquête sur l'immigration par l'Institut national des études démographiques (INED) intitulée «Mobilité géographique et insertion sociale» (MGIS). L’objectif explicite était d’analyser l'intégration des immigrés et de leurs descendants à la société française. À partir de ces données, plusieurs articles et ouvrages ont été publiés au milieu des années 1990 (Tribalat, 1995 ; Tribalat, Simon, Riandey, 1996). L’enquête comportait un échantillon de

I Pour une analyse comparée et critique dans la sociologie française des notions d'assimilation, d'intégration et d'insertion, voir Laacher, Sayad, 1998, et aussi dans ce numéro l'article de Jocelyne Streiff-Fénart. 
jeunes issus de plusieurs courants migratoires (Algérie, Portugal) ainsi que des questions sur l'expérience des discriminations. L'exploitation de ces données (Gaymu et Parant, 1996, p. 439-455 ; Simon, I998 p. 49-67) va soulever la question de la discrimination des enfants d'immigrés, notamment à l'entrée de la vie active. Dans la même période, l'exploitation approfondie d'une source statistique, l’Échantillon démographique permanent (EDP, INSEE), qui permet de repérer l'origine nationale des parents, conduit à poser aussi l'hypothèse d'une discrimination sur le marché du travail fondée sur l'origine migratoire (Richard, 1997). L'analyse des jeunes issus de l'immigration sortants du système éducatif (sans le baccalauréat) sur le marché du travail, à partir du panel téléphonique du CEREQ², «ancêtre » des enquêtes dites «Génération », aboutit à la même conclusion (Brinbaum et Werquin, 1997, p. 32-4I).

Depuis cette période, les études se sont multipliées (Brinbaum et Werquin, I999, p. I47-164, ibid., 2004, p. I45-166; Dupray et Moullet, 2004), encouragées par l'insertion de la lutte contre les discriminations dans l'agenda politique français à la fin des années 1990 (Lorcerie, I998, p. 69-8I). La plupart des travaux ont confirmé la pénalisation des enfants d'immigrés nord-africains dans l'accès à l'emploi en comparaison des Français d'origine mais aussi des descendants de l'immigration portugaise par exemple (Silberman et Fournier, 1999, p. 31-55) et ont avancé l'hypothèse d'une discrimination à base ethniste ou raciste ${ }^{3}$ sur le marché du travail.

Les travaux focalisés sur la transition à l'emploi des sortants de l'enseignement supérieur à partir des enquêtes du CEREQ, qui montraient des écarts entre enfants de l'immigration nord-africaine et enfants de l'immigration sud-européenne en début de vie active et une surexposition au chômage des premiers, ont notamment permis de repousser l'hypothèse selon laquelle ces situations d’inégalités n'étaient que la répercussion sur le marché du travail des écarts de formation (Frickey, Primon, 2002, p. 3I-49; Frickey, Murdoch,

2 Centre d'études et de recherches sur l'emploi et les qualifications.

3 L'expression «discrimination à base ethniste et raciste» est reprise de Véronique De Rudder, Christian Poiret et François Vourc'h (2000) et laisse entendre que ce type de discriminations est une matérialisation de rapports sociaux ethnistes ou racistes. 
Primon, 2004). L'hypothèse d'une assimilation segmentée a été clairement avancée dans une étude très riche réalisée par Patrick Simon, Dominique Meurs et Ariane Pailhé (2005) à partir des données du recensement de 1999 et aussi par Roxane Silberman et Irène Fournier (2006a et 2006b) qui s'appuient sur les enquêtes du CEREQ des années 1990 et qui montrent la pénalisation des enfants de nord-africains mais aussi d'Afrique sub-saharienne et de Turquie. Ces populations, toujours pénalisées dans les années 2000, sont d'ailleurs celles qui expriment davantage un sentiment de discrimination à l'embauche (Brinbaum et Guégnard, 20ı2a, p. 7-20). Ce modèle de l'assimilation segmentée est également discuté et avancé dans la thèse de Mirna Safi (2006) à partir des données de l'enquête MGIS.

Un des intérêts de l'enquête Trajectoire et Origines (TeO), dont sont tirés les résultats présentés dans cet article, est précisément de tenir compte des courants migratoires récents et de couvrir ainsi un spectre assez large d'origine migratoire à l'échelon national. Basée sur un sous-échantillon de descendants d'immigrés âgés de 18 à 35 ans qui ont terminé leur formation initiale, notre étude permet de revisiter les conditions d'entrée dans la vie active de ces populations et leur situation sur le marché du travail du début des années 1990 à la date de l'enquête (2008) avec l'hypothèse que le passage à la vie active et professionnelle ne s'effectue pas dans les mêmes conditions pour toutes les catégories de descendants d'immigrés, même si le niveau de formation et d’autres facteurs liés aux conditions sociales et familiales restent décisifs dans la détermination des trajectoires socio-professionnelles. En d'autres termes, les origines migratoires ne pèsent pas toutes d'un même poids dans la transition à l'emploi et certaines sont plus désavantagées que d'autres, en raison notamment d'une gestion différentielle des catégories de population sur le marché du travail et de l'existence de discriminations à caractères racistes qui barrent l'accès à l'emploi ou vulnérabilisent les groupes de population visés. Comme l'ont déjà montré plusieurs études, il y a tout lieu de penser que les descendants d'immigrés nord-africains, mais aussi ceux des pays d’Afrique sub-saharienne, se révèlent pénalisés.

Pour analyser la transition professionnelle et la situation sur le marché en 2008 des descendants d'immigrés, plusieurs descripteurs ont été retenus : 
- le comportement d’activité à la fin des études défini par le fait d’avoir connu une période d'activité (ou non) entre la fin des études et 2008;

- l'occupation d’un premier emploi dans l’année qui a suivi les études initiales ;

- le chômage à la date de l'enquête (2008) ;

- les formes de l'emploi à la date de l'enquête (2008).

Les deux premiers descripteurs ont été construits à partir d'une grille professionnelle indiquant année après année la situation d’activité des personnes. Sur cette base, seul le type d’activité est renseigné (inactivité, emploi salarié, emploi indépendants, etc.) et il n'est pas possible d’apprécier la qualité de l'emploi (salaire, temps de travail, forme d'emploi, etc.). Les deux derniers descripteurs font référence à la situation des actifs à la date de l'enquête. Ils proposent une mesure du chômage au sens du recensement et une description des conditions d’emploi centrée sur les formes de précarité au travail (contrats, temps partiel).

L'analyse qui suit se compose d'une première partie consacrée à la transition vers la vie professionnelle et d'une deuxième partie dédiée à la situation sur le marché du travail en 2008, à partir de l'examen du chômage et des formes d'emploi à la date de l'enquête des différentes catégories de descendants.

\section{L'enquête «Trajectoires et Origines» et le champ d'analyse}

En 2008, l'INED et l'INSEE ont réalisé une enquête nationale sur les immigrés et les descendants d'immigrés intitulée «Trajectoires et origines. La diversité des populations en France» ( $\mathrm{TeO})$.

Elle a été effectuée auprès de 22000 personnes âgées de 18 à 60 ans vivant dans un ménage ordinaire en France métropolitaine. Les descendants d'immigrés enquêtés sont âgés de 18 à 50 ans et les immigrés de 18 à 60 ans.

L'enquête vise à décrire et à analyser les conditions de vie et les trajectoires sociales des individus en fonction de leurs origines sociales et de leur lien à la migration. Elle analyse des populations issues de migrations variées, plus ou moins récentes, examine l'accès des individus aux biens et aux services (travail, logement, services, soins, etc.) ainsi que les discriminations pouvant $y$ faire obstacle.

Pour des informations complémentaires, voir: http://teo.site.ined.fr/ 


\begin{abstract}
Le champ d'analyse
La population étudiée comprend les personnes âgées de 18 à 35 ans ayant fini leurs études initiales classées parmi les descendants d'immigrés et dans la population dite «majoritaire » (ni immigrée ni issue de l'immigration). Les descendants d'immigrés sont tous nés en France et ont au moins un parent immigré, c'est-à-dire né à l'étranger et de nationalité étrangère. La plupart des descendants âgés de 18 à 35 ans sont de nationalité française (98\%). Les ressortissants de la population majoritaire sont des natifs en ce sens qu'ils sont nés en France de parents nés eux-mêmes Français en France. Les natifs des départements de l'outremer vivant en France métropolitaine et les descendants des ultramarins ne font pas partie du champ de l'analyse.
\end{abstract}

\title{
I. LA TRANSITION À LA VIE PROFESSIONNELLE
}

\section{I.I. LES DESCENDANTS D'IMMIGRÉS ET LEUR NIVEAU DE FORMATION}

À partir des données de l'enquête $\mathrm{TeO}$, on estime à un peu plus de 3 millions le nombre d'enfants d'immigrés en France en 2008 dont la moitié a moins de 30 ans (Borrel, Lhommeau, 20I0). Les descendants d'immigrés constituent une population particulièrement jeune, qui se compose pour moitié de personnes avec deux parents immigrés. Un tiers des descendants vivent en région parisienne.

Dans la sous-population âgée de I8 à 35 ans, les descendants d'immigrés européens se révèlent relativement âgés (40\% de plus de 30 ans contre $34 \%$ en moyenne) alors que les fils et filles de l'immigration turque s'avèrent beaucoup plus jeunes (18\% de 20 ans et moins contre $6 \%$ en moyenne). Les descendants d'immigrés subsahariens constituent aussi une population jeune avec I5 \% d'entre eux âgés de 20 ans et moins. Les fils et filles de l'immigration du sud-est asiatique sont à peine plus vieux avec plus de $43 \%$ de 25 ans ou moins (32\% en moyenne) alors que ceux d'immigrés algériens ou portugais se situent dans la moyenne. Les plus nombreux sont les descendants de l'immigration européenne qui représentent $24 \%$ de la tranche d'âge. Ils sont suivis de près par les fils et filles de l'immigration algérienne qui comptent pour $22 \%$. Au total, les descendants des immigrés nord-africains représentent $40 \%$ des descendants d'immigrés âgés de 18 à 35 ans. Une autre catégorie qui pèse 
quantitativementest formée des fils et filles d'immigrés portugais ( $17 \%$ ). Les autres origines migratoires, malgré leur jeunesse, constituent des groupes relativement marginaux: Afrique subsaharienne (5\%); descendants d'immigrés turcs ou d'Asie du sud-est (3\%).

\section{Le regroupement des origines migratoires et géographiques}

Dans les analyses, les origines migratoires des personnes sont classées par pays ou par grandes régions de naissance des parents en tenant compte de la structure de l'échantillon de l'enquête TeO. Deux nomenclatures de publication sont utilisées: une classification détaillée en neuf postes et une agrégée en huit postes.

Classification détaillée des origines migratoires et géographiques (9 postes):

I. Population majoritaire: ni immigrés, ni descendants d'immigrés.

2. Descendants d'immigrés originaires d'Algérie.

3. Descendants d'immigrés originaires du Maroc ou Tunisie.

4. Descendants d'immigrés originaires des pays d'Afrique subsaharienne (hors Afrique de l'Est et australe).

5. Descendants d'immigrés originaires de Turquie.

6. Descendants d'immigrés originaires d'Asie du sud-est: Vietnam, Cambodge, Laos.

7. Descendants d'immigrés originaires du Portugal.

8. Descendants d'immigrés originaires des pays de l'Union européenne à 27 (autres que le Portugal et la France).

9. Descendants d'immigrés originaires d'autres pays.

L'agrégation en huit postes se fait en regroupant les descendants d'immigrés originaires d'Algérie, du Maroc ou de Tunisie en une seule catégorie: les descendants d'immigrés originaires d'Afrique du Nord.

Les niveaux de formation et scolaires sont très variables selon les origines migratoires des descendants d'immigrés. ${ }^{4}$ Dans la population âgée de I 8 à 35 ans, en comparaison de la population majoritaire, des niveaux particulièrement bas sobservent parmi les descendants des immigrés originaires de Turquie qui se composent de $36 \%$ de non diplômés ou de titulaires d'un simple brevet des collèges. Au mieux, I5 \% d'entre eux ont acquis un diplôme de l'enseignement 
supérieur (court ou long) contre $40 \%$ dans la population générale. Le niveau de diplôme des descendants d'immigrés originaires d’Algérie demeure également faible avec I $8 \%$ de sans-diplôme et I I \% de titulaires du brevet pour seulement $23 \%$ de diplômés du supérieur court ou long (40\% dans la population majoritaire). Les descendants d'immigrés du Maroc / Tunisie se révèlent très proches des descendants d'Algériens avec cependant moins de sans-diplôme. Les descendants d'immigrés originaires des pays d'Afrique subsaharienne se composent également de nombreux non diplômés (26\% sans aucun diplôme ou avec un brevet) avec un déficit de diplômés du supérieur (27\%).

À l'opposé de ces groupes, se trouvent la population majoritaire, mais aussi les descendants de l'immigration européenne (hors Portugal) dont la répartition dans les niveaux de diplôme est sensiblement la même que celle de la population majoritaire. Les descendants d'immigrés originaires du sud-est asiatique forment quant à eux un groupe au niveau scolaire très spécifique en ce sens que la part des diplômés de l'enseignement supérieur (court ou long) y représente la moitié de la catégorie. Les descendants d’immigrés dont l'origine est regroupée dans les «autres pays » se remarquent aussi par un niveau très élevé, mais leur provenance géographique étant extrêmement hétérogène, leurs résultats ne seront pas commentés. Reste les descendants de l'immigration portugaise qui se démarquent des autres groupes d'origine par le poids des détenteurs d'un CAP / BEP (33\% contre $23 \%$ en moyenne) et l'investissement dans les diplômes professionnels.

Il va de soi que cette structure très inégale des niveaux scolaires et le fait que plusieurs catégories de population se composent d'un pourcentage élevé de non diplômés pèsent sur la transition à la vie active et la position sur le marché du travail, compte tenu du rôle toujours déterminant du diplôme dans le passage de l'école à l'emploi. D’autres facteurs socio-démographiques comme les origines sociales et familiales sont susceptibles d'interférer sur la relation à l'emploi : plusieurs catégories de descendants d'immigrés sont issues de familles d'une origine sociale extrêmement populaire où la mère est très souvent inactive. 


\section{I.2. ENTRER DANS LA VIE ACTIVE \\ Une causalité du probable?}

Parmi les jeunes âgés de I8 à 35 ans ayant terminé leurs études, $6 \%$ n’ont jamais été actifs, c'est-à-dire en emploi ou au chômage. ${ }^{5}$ Après la fin de la formation initiale, l'entrée dans la vie professionnelle et la mise au travail constituent la destinée de la plupart des jeunes (femmes ou hommes), seule une petite minorité (au sens numérique) n'expérimente pas la vie active (voir Figure I).

En l'occurrence, les inactifs sont plutôt des inactives ( $8 \%$ de femmes inactives) et les plus jeunes (un quart des moins de 20 ans). Ces derniers ont quitté depuis peu le système éducatif en étant très souvent faiblement diplômés. Cette inactivité juvénile pourrait cependant ne pas être durable et le passage à l'activité différé à une date ultérieure.

Pour autant, indépendamment de l'âge atteint en 2008 et de l'ancienneté de la fin des études, les jeunes de 18 à 35 ans sans diplôme (en deçà du brevet) sont relativement nombreux à n'avoir aucune expérience du travail (i6\%). Malgré le caractère généralisé du passage à l'activité (94\%), la démarche n’a rien d’automatique dans les fractions les plus démunies scolairement et se destiner ou non à la vie professionnelle reste fortement conditionné par le diplôme.

En effet, tendanciellement ce sont les diplômés disposant des chances objectives d'accès à l'emploi les plus faibles qui affichent la propension à l'inactivité la plus forte (voir Figure I) ; le taux d'inactivité et le taux des actifs en emploi à la fin des études initiales évoluant en raison inverse. Entrer dans la vie active (ou ne pas y entrer) s'apparente collectivement à une causalité du probable où les conduites se règlent sur le devenir objectif (Bourdieu, 1974). L'inactivité peut dès lors être lue comme une position de retrait de la vie professionnelle qui anticipe une sélection négative sur le marché du travail. L’inactivité, au sens du travail marchand, ne veut pas dire passivité, mais une orientation préférentielle vers l'ordre domestique et familial et le maintien dans une situation de 
dépendance économique voire, le cas échéant, une participation à des formes d’activités informelles ou illégales que l'enquête ne permet pas de repérer.

Figure I. Inactivité en cours de vie et emploi après les études des I8-35 ans selon le diplôme (\%)

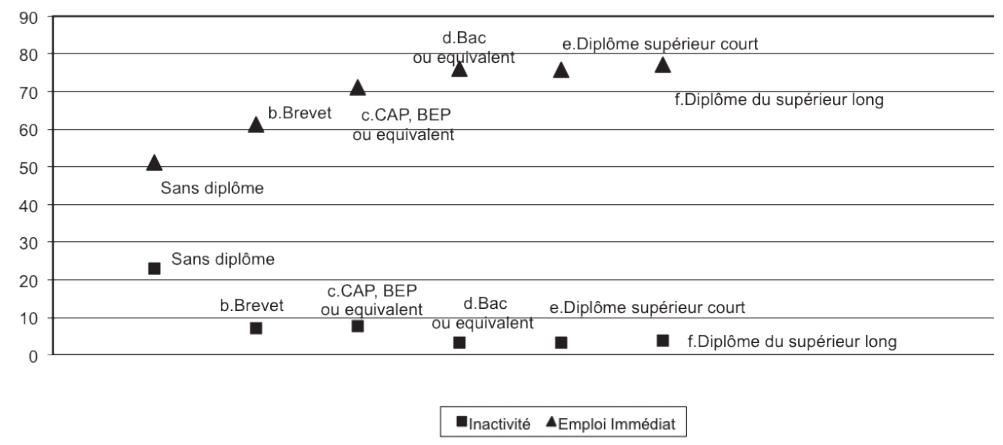

Source: enquête «Trajectoires et Origines», 2008, Ined-Insee.

Champ: jeunes âgés de 18 à 35 ans en 2008 et ayant achevé leur formation initiale.

Lecture: On décompte parmi les sans diplômes plus de 20\% d'inactifs pour un taux d'actifs en emploi dans l'année consécutive à la sortie de formation de $50 \%$.

Parmi les jeunes non diplômés, qui ont quitté les études après le collège, le poids de l'inactivité représente $14 \%$ chez les hommes et $18 \%$ chez les femmes. Chez les non diplômés de sexe masculin, l'inactivité concerne très peu les descendants d'immigrés européens (5\%), en revanche elle est nettement plus répandue parmi les descendants de nord-africains et subsahariens (16\%), mais aussi dans la population majoritaire (15\%). Les sorties sans diplôme de ces populations résultent d'un cumul d'inégalités sociales et familiales, mais aussi d'orientations contrariées subies dans les filières professionnelles qui aboutissent à des échecs et pénalisent l'entrée dans la vie active (Brinbaum, Guégnard, 20I2b, p. 6I-82).

Parmi les femmes non diplômées, celles appartenant à la population majoritaire se révèlent souvent inactives (19\%). Le taux d’inactivité diminue sensiblement parmi les descendantes d’immigrés : I5\% pour les descendantes d'immigrés nord-africains ou subsahariens; i3 \% chez celles originaires des pays européens. 
Les résultats plus détaillés (non communiqués) selon les origines des moins diplômés (brevet et en deçà) montrent, en revanche, des taux d'inactivité sensiblement plus élevés que la moyenne (18\%) dans deux catégories de descendantes d'immigrés : celles originaires des pays d'Afrique sub-saharienne (27\%) et celles originaires de Turquie (30\%). Dans la population masculine, les jeunes originaires d'Afrique subsaharienne ne se distinguent pas des autres groupes, en revanche les jeunes hommes originaires de Turquie se révèlent plus actifs que ceux de la population majoritaire. Chez les descendants de turcs, la différenciation du comportement d'activité entre hommes et femmes s'avère très marquée : le taux d'inactivité féminine étant le plus élevé de toutes les catégories. Pour autant toutes les jeunes femmes d'origine turque ne se trouvent pas hors du marché du travail, néanmoins, comme va le voir ci-après, la conversion au travail marchand, pour elles, se révèle particulièrement difficile, faute sans doute de disposer d'un modèle d'activité maternelle de référence en la matière et d'un capital relationnel professionnel sur lequel s'appuyer.

Figure 2. Inactivité en cours de vie et emploi après les études des I8-35 ans selon l'origine migratoire (\%)

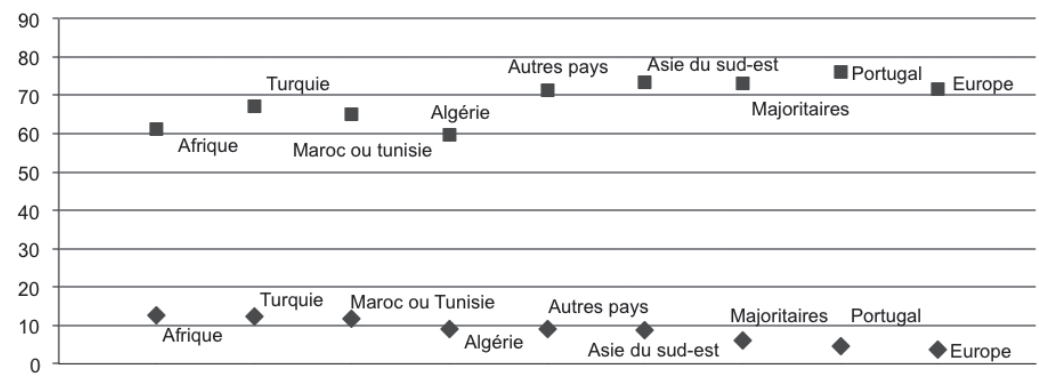

•Inactivité Emploi immédiat

Source: enquête «Trajectoires et Origines», 2008, Ined-Insee.

Champ: jeunes âgés de 18 à 35 ans en 2008 et ayant achevé leur formation initiale.

Lecture: On décompte parmi les descendants d'immigrés d'Afrique subsaharienne plus de $10 \%$ d'inactifs pour un taux d'actifs en emploi dans l'année consécutive à la sortie de formation initiale de 60\%. 
Dans l'ensemble, la corrélation négative entre inactivité et emploi rapide vaut aussi selon les origines migratoires: plus le taux d'emploi immédiat augmente et plus l'inactivité diminue (voir Figure 2). Mais il y a visiblement des exceptions. Les descendants d'immigrés algériens en constituent l'exemple le plus éloquent : bien que figurant parmi les plus défavorisés relativement aux chances d'être immédiatement en emploi au terme de la formation initiale, les descendants d'algériens ne sont pas pour autant les plus inactifs. Dans une moindre mesure, ce décalage est visible aussi pour les descendants d'immigrés du Maroc ou de Tunisie. Cette relation à l'activité de la descendance de l'immigration algérienne, marque d'une bonne volonté professionnelle, est largement imputable au comportement d'activité de la population féminine (voir Figure 3). Les descendantes de l'immigration algérienne, bien que plus rarement en emploi immédiatement après les études que les descendantes d’Afrique sub-saharienne ou du Maroc et Tunisie, par exemple, n'en sont pas plus inactives (ı\%): c'est là le signe tangible d'une conversion historique à l'activité marchande et salariale de ces jeunes femmes qui rompt avec le modèle de l'inactivité maternelle encore très répandu dans les familles de l'immigration algérienne. Cas inverse : les descendantes de l'immigration du sud-est asiatique qui, tout en affichant un taux d'emploi immédiat parmi les plus élevés (76\%), restent en moyenne fortement inactives (i \% \%). Dans la population féminine, la logique d'une causalité du probable n'épuise pas le rapport à l'activité des différentes catégories de descendantes et d'autres facteurs que le niveau de diplôme ou la situation probable sur le marché du travail entrent dans la détermination des comportements d'activité. 
Figure 3. Inactivité en cours de vie et emploi après les études des i 8-35 ans dans la population féminine selon l'origine migratoire (\%)

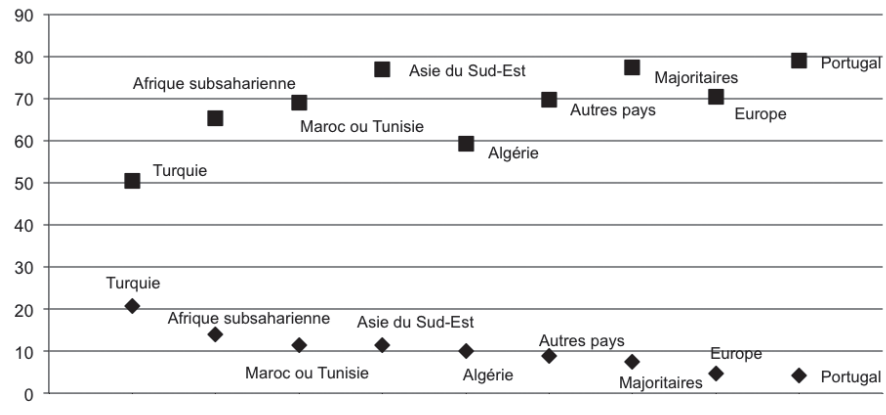

- Inactivité $\mathbf{m}$ Emploi immédiat

Source: enquête «Trajectoires et Origines», 2008, Ined-Insee.

Champ: jeunes femmes âgées de 18 à 35 ans en 2008 et ayant achevé leur formation initiale.

Lecture: On décompte parmi les descendantes d'immigrés turcs 20\% d'inactives pour un taux d'actives en emploi dans l'année consécutive à la sortie de formation initiale de 50\%.

\section{I.3. L'ACCÈS AU PREMIER EMPLOI}

$84 \%$ des actifs $^{6}$ entrent dans la vie active la même année que le terme de la formation initiale et $72 \%$ y accèdent directement par l'emploi. Les données de $\mathrm{TeO}$ ne nous permettent pas d'évaluer la qualité du premier emploi occupé mais nous renseignent sur la célérité de l'entrée dans la vie professionnelle. Pour les deux tiers des jeunes de 18 à 35 ans, le temps de latence entre l'entrée dans la vie active et l'entrée dans la vie professionnelle est inexistant ou n'excède pas l'année. Comme pour le comportement d’activité, le niveau de diplôme conditionne les chances d’accéder rapidement à l'emploi : seuls 51 \% des actifs non-diplômés entrent directement dans l'emploi contre $75 \%$ et plus pour les bacheliers et les diplômés du supérieur. Le niveau de diplôme constitue une bonne garantie pour occuper un emploi dans l’année consécutive aux études.

6 Dans les traitements, sont classés parmi les actifs, les individus ayant connu au mois une période d'activité depuis la fin des études. 
Au regard de la vitesse d'accès au premier emploi, il existe de grandes disparités selon les origines migratoires: dans le groupe majoritaire, $73 \%$ des actifs accèdent immédiatement à l'emploi après la formation initiale, mais ils ne sont que $60 \%$ parmi les descendants d’immigrés algériens et 6I \% chez ceux de l'immigration subsaharienne.

Les inégalités entre origines migratoires se retrouvent aussi à l'intérieur des groupes de sexe. Dans l'ensemble, les femmes accèdent au premier emploi (76\%) dans des proportions plus grandes que les hommes (69\%) en raison principalement d'un niveau de formation plus élevé que ces derniers, excepté parmi les fils et filles d'immigrés turcs où le rapport hommes-femmes est inversé. Au sein de la population féminine, les écarts entre les origines migratoires s'avèrent souvent très importants. Ainsi, $77 \%$ des jeunes femmes de la population majoritaire entrent dans la vie active en occupant un emploi alors que seules $59 \%$ des descendantes de l'immigration algérienne et 50\% des descendantes de l'immigration turque y parviennent. Chez les hommes, toujours au regard de la célérité dans l'accès au premier emploi, les descendants d’immigrés algériens (60\%), du Maroc ou de Tunisie (6I \%) et plus encore des pays d'Afrique subsaharienne ( $57 \%$ ) apparaissent particulièrement désavantagés en comparaison de la population majoritaire (69\%). En contrepartie, on peut noter parmi les femmes actives, que les descendantes de l'immigration portugaise (79\%) ou d’Asie du Sud-Est (77\%) accèdent rapidement au premier emploi. Dans la population masculine, sous ce rapport, nous retrouvons les mêmes catégories de descendants que dans la population féminine (sud-est asiatique : $70 \%$; Portugal : 74\%) auxquelles s'ajoutent les descendants des pays européens (72\%) et plus encore ceux originaires de Turquie (79\%). Au regard des modes d'entrée dans la vie professionnelle, force est de constater que les destins ne se ressemblent pas selon les origines migratoires mais que, pour une origine donnée, la célérité d’accès à l'emploi se révèle différenciée selon les sexes.

Les inégalités scolaires qui séparent les catégories de descendants et descendantes comptent pour beaucoup dans les voies divergentes qu'empruntent les destinées professionnelles. Or, la prise en considération des héritages sociaux et familiaux dans les modélisations de l'accès au premier emploi (voir Tableau I), 
montre que plusieurs facteurs socio-démographiques contribuent à expliquer les écarts observés selon les origines migratoires.? Malgré tout, certaines provenances paraissent plus désavantagées que d'autres. En effet, dans la population masculine comme féminine, les descendants d'immigrés nord-africains et d'Afrique subsaharienne connaissent des chances moindres d'occuper directement un emploi après les études en comparaison de la population majoritaire. Même lorsque l'âge et le niveau de diplôme sont contrôlés (voir Tableau I, modèle 2), le désavantage persiste, alors que pour les deux sexes le fait de ne pas avoir de diplôme (ou d'être faiblement diplômé) agit négativement sur l'accès au premier emploi. Dans la population féminine, la détention du baccalauréat ou de diplômes du supérieur augmente a contrario la probabilité d'occuper un premier emploi rapidement. Chez les hommes, avoir entre 30 et 35 ans semble plutôt avoir freiné l'accès au poste, sans doute à cause d'une conjoncture économique défavorable à la sortie du système éducatif dans la première moitié des années 1990, mais aussi en raison de l'existence d'un service national qui obligeait les jeunes hommes à reporter la prise d'emploi. L’accès à l'emploi est également dépendant, dans les deux groupes de sexe, des inégalités territoriales: le fait de résider dans une zone urbaine sensible, où généralement l'emploi manque, handicape la mise au travail rapide; à l'opposé une résidence parisienne constitue un avantage objectif en raison des opportunités offertes par le marché du travail. Enchaîner les études et la vie professionnelle n'est pas uniquement une question de forma-

7 Pour approfondir l'analyse, plusieurs modèles de régression logistique ont été mis en œuvre afin d'expliquer les écarts observés entre les origines migratoires et la population majoritaire, en prenant en compte des caractéristiques socio-démographiques ou socio-économiques (autres que l'origine migratoire) susceptibles d'influer sur la transition professionnelle ou la situation sur le marché du travail. Les populations masculine et féminine ont été analysées séparément en raison de la forte différenciation que l'on observe dans leurs trajectoires scolaires et professionnelles. Dans les modèles, la mesure de l'inégalité est donnée par le «odds ratio » (OR) - traduit souvent en français par «rapport des chances»-qui représente le ratio entre deux probabilités: par exemple la probabilité d'être actif et son complément (être inactif) ou encore la probabilité d'être chômeur (ou non). Une mesure supérieure à l'unité $(O R>1)$ signifie que la probabilité d'être actif est plus élevée que la probabilité contraire. Inversement, si $O R<1$, la probabilité étudiée est plus faible. OR vaut I en situation d'indépendance statistique entre la variable explicative et la variable d'intérêt. Aucune pondération n'a été appliquée dans les modélisations. 
tion ou d'offre d'emplois, mais aussi de distance plus ou moins grande des familles au monde du travail. Chez les hommes comme chez les femmes, le fait d'avoir ses deux parents en dehors de la sphère de travail à l'âge de la fin des études pèse lourd sur les chances d'obtenir un emploi rapidement, faute de pouvoir activer des liens familiaux ou personnels favorisant l'embauche. On mesure, à travers ces situations, l'action négative sur la descendance de la marginalisation socio-économique des parents engendrée par les restructurations industrielles et les nouvelles règles de l'ordre économique. Pour autant, même en prenant en considération ces facteurs déterminants, le désavantage dans l'accès à l'emploi des descendants d'immigrés subsahariens des deux sexes ou des descendantes de nord-africains ne disparaît pas. Comme dans les études antérieures, tout porte à croire que les descendants de ces origines migratoires, que l'on sait par ailleurs être couramment stigmatisées et qui déclarent souvent faire l'expérience de discriminations à base ethnistes ou racistes, se heurtent à la sortie des études et sur le marché du travail à des barrages spécifiques et à un traitement différentiel.

La place des descendants d'immigrés turcs reste particulière car ce groupe affiche une profonde division des rôles de sexe dans la relation à l'emploi avec des hommes qui sont pour ainsi dire sur-occupés, et cela malgré leur faible niveau scolaire. Tout se passe comme si l'interruption précoce de leur scolarisation se justifiait par des opportunités d'emploi, sans doute générées par les liens de familles. Le poids des positions indépendantes dans la structure des emplois occupés par les hommes descendants de l'immigration turque (I4.6\%) tend à faire valoir une conversion incomplète de cette population à la condition salariale et aussi la survivance d'un mode de reproduction sociale fondé directement sur l'héritage économique plutôt que sur les classements scolaires. Les descendantes de l'immigration turque, en revanche, peinent à trouver du travail lorsque leur trajectoire s'écarte du modèle maternel où prédomine l'inactivité. 
Tableau I. Modélisation de l'accès rapide au premier emploi en début de vie active dans la population masculine et féminine (odds ratios)

\begin{tabular}{|c|c|c|c|c|c|c|c|c|c|c|c|c|c|}
\hline & & \multicolumn{6}{|c|}{ POPULATION MASCULINE } & \multicolumn{6}{|c|}{ POPULATION FÉMININE } \\
\hline & & \multicolumn{2}{|c|}{ Modèle I } & \multicolumn{2}{|c|}{ Modèle 2} & \multicolumn{2}{|c|}{ Modèle 3} & \multicolumn{2}{|c|}{ Modèle I } & \multicolumn{2}{|c|}{ Modèle 2} & \multicolumn{2}{|c|}{ Modèle 3} \\
\hline \multirow{8}{*}{$\begin{array}{c}\text { ORIGINE } \\
\text { MIGRATOIRE }\end{array}$} & $\begin{array}{l}\text { Population } \\
\text { majoritaire }\end{array}$ & ref. & & ref. & & ref. & & ref. & & ref. & & ref. & \\
\hline & $\begin{array}{l}\text { Afrique du } \\
\text { Nord }\end{array}$ & 0,68 & ***** & 0,73 & $* *$ & 0,80 & & 0,59 & ***** & 0,705 & ***** & 0,74 & **⿻丷木 \\
\hline & $\begin{array}{l}\text { Afrique sub- } \\
\text { saharienne }\end{array}$ & 0,61 & 米米类 & 0,62 & $* *$ & 0,66 & $* *$ & 0,61 & ****** & 0,67 & *** & 0,59 & ****** \\
\hline & $\begin{array}{l}\text { Asie du } \\
\text { Sud-Est }\end{array}$ & 0,99 & & 0,83 & & 0,87 & & $\mathrm{I}, 00$ & & I,06 & & 1,05 & \\
\hline & Turquie & 1,72 & $* *$ & 1,87 & **** & 2,03 & ***** & 0,41 & ***** & 0,62 & ** & 0,70 & \\
\hline & Portugal & 1,23 & & 1,31 & & 1,26 & & 0,99 & & 1,02 & & 0,96 & \\
\hline & $\begin{array}{l}\text { Europe (hors } \\
\text { Portugal) }\end{array}$ & 1,05 & & I,II & & 1,10 & & 0,82 & & 0,85 & & 0,84 & \\
\hline & Autres pays & 1,05 & & 1,03 & & 1,06 & & 0,73 & & 0,66 & *** & 0,59 & ******* \\
\hline \multirow{4}{*}{$\begin{array}{c}\text { ÂGE EN } \\
2008\end{array}$} & $\begin{array}{l}20 \text { ans et } \\
\text { moins }\end{array}$ & & & 0,89 & & 0,88 & & & & 0,86 & & 0,86 & \\
\hline & $\begin{array}{l}25 \text { ans et } \\
\text { moins }\end{array}$ & & & ref. & & ref. & & & & ref. & & ref. & \\
\hline & $\begin{array}{l}30 \text { ans et } \\
\text { moins }\end{array}$ & & & 1,18 & & 1,18 & & & & 0,91 & & 0,90 & \\
\hline & $\begin{array}{l}35 \text { ans et } \\
\text { moins }\end{array}$ & & & 0,55 & ***** & 0,53 & ***** & & & 0,99 & & 0,98 & \\
\hline
\end{tabular}


Revue européenne des sciences sociales

\begin{tabular}{|c|c|c|c|c|c|c|c|c|c|}
\hline \multirow{6}{*}{ DIPLÔME } & $\begin{array}{l}\text { Aucun } \\
\text { diplôme }\end{array}$ & 0,46 & 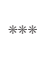 & 0,48 & ***** & 0,35 & **** & 0,36 & **** \\
\hline & Brevet & 0,54 & ****** & 0,55 & **** & 0,50 & **** & 0,50 & ***** \\
\hline & $\begin{array}{l}\text { CAP,BEP ou } \\
\text { équivalent }\end{array}$ & ref. & & ref. & & ref. & & ref. & \\
\hline & Baccalauréat & 1,14 & & 1,13 & & 1,00 & & 0,97 & \\
\hline & $\begin{array}{l}\text { Diplôme du } \\
\text { supérieur } \\
\text { court } \\
(\mathrm{bac}+2)\end{array}$ & 0,97 & & 0,96 & & 1,94 & **** & 1,81 & **** \\
\hline & $\begin{array}{l}\text { Diplôme du } \\
\text { supérieur } \\
\text { long }\end{array}$ & 1,19 & & 1,19 & & 2,07 & **** & 1,79 & **** \\
\hline \multirow{4}{*}{$\begin{array}{c}\text { ORIGINE } \\
\text { SOCIALE } \\
\text { DES DEUX } \\
\text { PARENTS }\end{array}$} & $\begin{array}{l}\text { Agriculteurs, } \\
\text { artisans et } \\
\text { commerçants }\end{array}$ & & & 1,16 & & & & 0,93 & \\
\hline & $\begin{array}{l}\text { Cadres et } \\
\text { prof, sup, \& } \\
\text { prof. intermé- } \\
\text { diaires }\end{array}$ & & & 0,86 & & & & 1,06 & \\
\hline & $\begin{array}{l}\text { Ouvriers et } \\
\text { employés } \\
\text { non qualifiés }\end{array}$ & & & & & & & ref & \\
\hline & $\begin{array}{l}\text { Ouvriers et } \\
\text { employés } \\
\text { qualifiés }\end{array}$ & & & 1,04 & & & & 0,95 & \\
\hline \multirow{3}{*}{$\begin{array}{l}\text { ACTIVITÉ } \\
\text { DES DEUX } \\
\text { PARENTS }\end{array}$} & $\begin{array}{l}\text { Deux parents } \\
\text { au travail }\end{array}$ & & & 1,09 & & & & 1,06 & \\
\hline & $\begin{array}{l}\text { Deux parents } \\
\text { hors travail }\end{array}$ & & & 0,67 & *** & & & 0,58 & **** \\
\hline & $\begin{array}{l}\text { Un seul } \\
\text { parent au } \\
\text { travail }\end{array}$ & & & ref. & & & & ref. & \\
\hline
\end{tabular}




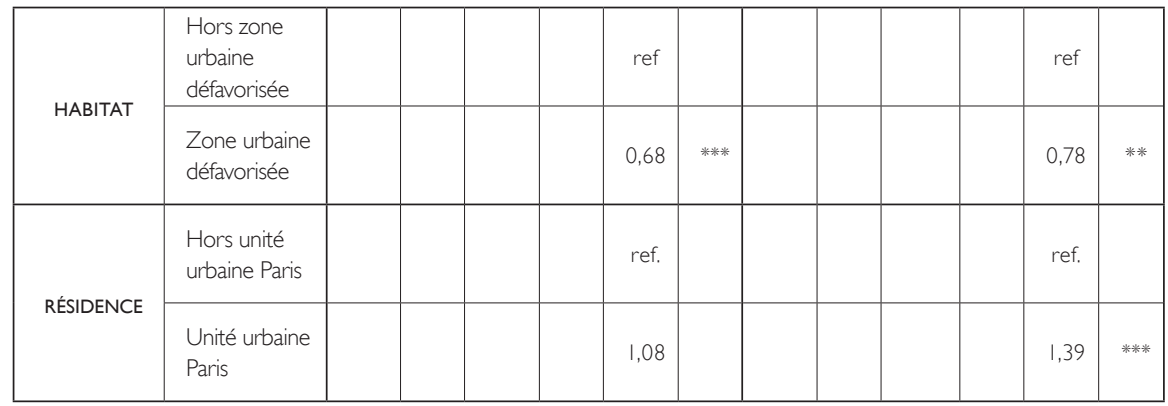

Les seuils de significativité sont respectivement égaux à I \% (***), $5 \%$ (**) et $10 \%(*)$.

Source: enquête «Trajectoires et Origines», 2008, Ined-Insee.

Champ: jeunes actifs âgés de 18 à 35 ans en 2008 ayant achevé leur formation initiale.

Lecture: Parmi les descendants masculins d'immigrés nord-africains actifs, la probabilité d'occuper un emploi immédiatement après les études (plutôt que plus tard) ou jamais en comparaison de la population majoritaire masculine s'élève à 0,68 ; ce qui signifie que la probabilité d'un emploi rapide (plutôt que plus tard ou jamais) pour les premiers est 1,46 fois moins élevée que chez les seconds.

\section{CHÔMAGE ET FORMES D’EMPLOI}

\section{I. UN SUR-CHÔMAGE POUR CERTAINES ORIGINES MIGRATOIRES}

La pénalisation de certaines catégories de descendants d'immigrés (Afrique du Nord et Afrique subsaharienne) déjà visible dans la transition à la vie active se répète avec la mesure du chômage à la date de l'enquête.

En 2008, la part des jeunes actifs de I8 à 35 ans sans emploi ou en recherche d'emploi - autrement dit chômeurs - varie significativement selon l'origine migratoire et le sexe (voir Tableau 2). Comparés à la population majoritaire (I3,4 \% de chômage), les taux de chômage ${ }^{8}$ apparaissent deux à trois fois supérieurs chez les hommes et les femmes descendants de l'immigration nord-

8 Le taux de chômage est calculé au sens du recensement à partir de plusieurs variables présentes dans l'enquête $\mathrm{TeO}$ : la situation principale vis-à-vis du travail, le fait de travailler à la date de l'enquête et de rechercher ou non un emploi. Ainsi, le taux de chômage mesure-t-il le nombre de personnes se déclarant au chômage et en recherche d'emploi rapporté au nombre d'actifs (personnes en emploi ou en recherche d'emploi). 
africaine (avec respectivement $26 \%$ et $24 \%$ de chômage). Le taux de chômage s'avère aussi très élevé chez les hommes originaires d’Afrique subsaharienne (32\%) et d'Asie du Sud Est (23\%). Sous le rapport du chômage, ces deux catégories de descendants se caractérisent par ailleurs par des écarts de sexe très élevés en défaveur de la population masculine alors que la tendance est plutôt inversée dans la population majoritaire : faut-il y voir un effet de la dimension sexuée des inégalités scolaires? Ou bien le traitement des sexes pour ces origines migratoires est-il différencié sur le marché du travail?

La population descendante de l'immigration turque affiche aussi des taux de chômage particulièrement hauts, surtout dans la population féminine, avec respectivement $25 \%$ de chômage chez les hommes, mais $38 \%$ chez les femmes. À l'instar de la mise en activité ou de l'accès au premier emploi, face au chômage les descendantes de l'immigration turque apparaissent particulièrement désavantagées. À l'opposé de ces catégories particulièrement vulnérables au chômage, dont le quart, voire le tiers, de la population expérimente le chômage, les taux s'avèrent très faibles pour les descendants d'immigrés portugais (et cela est particulièrement vrai chez les femmes) et parmi les descendants des autres pays de l’Union Européenne. Dans ces deux catégories, les taux de chômage sont même inférieurs à ceux observés dans la population majoritaire. 
Tableau 2. Taux de chômage en 2008 selon l'origine migratoire et le sexe

\begin{tabular}{|l|c|c|c|}
\hline & HOMMES (\%) & FEMMES (\%) & ENSEMBLE (\%) \\
\hline POPULATION MAJORITAIRE & 12,4 & 14,1 & 13,2 \\
\hline AFRIQUE DU NORD & 26,0 & 23,8 & 24,9 \\
\hline AFRIQUE SUBSAHARIENNE & 32,0 & 17,6 & 24,9 \\
\hline ASIE DU SUD-EST & 23,0 & 12,1 & 18,1 \\
\hline TURQUIE & 24,9 & 38,2 & 29,7 \\
\hline PORTUGAL & 8,2 & 4,4 & 6,6 \\
\hline EUROPE (HORS PORTUGAL) & 7,3 & 9,0 & 8,1 \\
\hline AUTRES PAYS & 11,5 & 13,0 & 12,1 \\
\hline
\end{tabular}

Source: enquête «Trajectoires et Origines», 2008, Ined-Insee.

Champ: actifs âgés de 18 à 35 ans (ayant fini leurs études initiales).

Lecture: dans la population majoritaire, chez les hommes actifs, le taux de chômage s'élève à 12,4\%; dans la population féminine, il atteint $14,1 \%$.

Les écarts de chômage à la fois entre les groupes et entre les sexes au sein des groupes masquent des effets imputables à la structure différentielle des populations: écarts d’âge, niveaux de diplôme, origines sociales, etc. En vue de tenir compte de ces effets de structure et de démêler les effets des origines migratoires et des autres facteurs sociodémographiques, nous avons estimé la probabilité d'être au chômage, séparément pour les hommes et pour les femmes, en fonction de l'origine migratoire et d'autres facteurs explicatifs.

$\mathrm{Au}$ vu des résultats (voir Tableau 3), il est notable que sur le marché du travail, les plus jeunes sont les plus vulnérables avec une probabilité de chômage deux fois plus élevée que pour les âges intermédiaires (25-30 ans). Arrivés depuis peu sur le marché du travail, ils cumulent à la fois le manque d'expérience et a fortiori de relations professionnelles, et leur situation dépend plus étroitement que les plus âgés de la conjoncture économique, en particulier en périodes de crise. Les plus jeunes parmi les actifs sont aussi les moins diplômés. 
Les sans diplômes sont toujours les plus pénalisés et connaissent les plus fortes probabilités de chômage ( $\mathrm{OR}=2,6$ pour les femmes et 2,8 pour les hommes). La résidence dans une zone urbaine défavorisée augmente aussi la probabilité de chômage - toutes choses étant égales par ailleurs - chez les jeunes hommes ; chez les jeunes femmes, c'est une résidence parisienne qui constitue un avantage.

Mais le résultat le plus saillant se trouve dans le fait que, même avec des caractéristiques socio-démographiques semblables, l'effet de l'origine migratoire sur le chômage (en comparaison de la population majoritaire) ne disparaît pas chez les jeunes hommes et femmes originaires des pays d'Afrique du Nord. Dans cette catégorie de descendants, la surexposition au chômage concerne les deux sexes. En revanche, parmi les descendants de l'immigration subsaharienne ou du sud-est asiatique, le sur-chômage est une expérience masculine et non féminine. Nous observons la situation inverse dans la population originaire de Turquie avec une très grande vulnérabilité au chômage des jeunes femmes mais non des jeunes hommes, toutes choses étant égales par ailleurs.

À l'opposé de ces trajectoires de chômage, conséquence pratique d'une gestion différentielle des catégories de population sur la base de l'origine migratoire et du sexe, se trouvent les descendants de l'immigration portugaise des deux sexes qui, même à caractéristiques identiques, expérimentent moins le chômage que les autres catégories (en comparaison de la population majoritaire) conformément aux résultats des études antérieures. À un degré moindre, la même observation peut-être faite pour les descendants des immigrations européennes. Il est intéressant de noter que les populations les moins exposées au discriminations parmi les catégories de descendants d'immigrés (Beauchemin et al., 20ı; Brinbaum et al., 20ı) sont aussi celles qui sont les moins vulnérabilisées socio-économiquement. 
Tableau 3: La probabilité d'être au chômage en 2008 par sexe (Odds ratios)

\begin{tabular}{|c|c|c|c|c|c|c|c|c|c|}
\hline & & \multicolumn{4}{|c|}{ HOMMES } & \multicolumn{4}{|c|}{ FEMMES } \\
\hline & & \multicolumn{2}{|c|}{ Modèle I } & \multicolumn{2}{|c|}{ Modèle 2} & \multicolumn{2}{|c|}{ Modèle I } & \multicolumn{2}{|c|}{ Modèle 2} \\
\hline \multirow{8}{*}{ ORIGINE MIGRATOIRE } & Population majoritaire & ref. & & ref. & & ref. & & ref. & \\
\hline & Afrique du Nord & 2,30 & **** & 1,89 & **** & 1,92 & **** & 1,69 & **** \\
\hline & Afrique subsaharienne & 2,98 & **** & 1,66 & $* *$ & 1,45 & * & 1,22 & \\
\hline & Asie du Sud-Est & 2,14 & ***** & 1,67 & $*$ & 0,92 & & 0,70 & \\
\hline & Turquie & 1,47 & & 0,79 & & 3,66 & ***** & 1,91 & *** \\
\hline & Portugal & 0,59 & $* *$ & 0,51 & **** & 0,42 & **** & 0,47 & *** \\
\hline & Europe (Hors Portugal) & 0,58 & *** & 0,66 & $*$ & 0,66 & * & 0,69 & \\
\hline & Autres pays & 0,98 & & 0,93 & & 0,98 & & 1,10 & \\
\hline \multirow{4}{*}{ ÂGE EN 2008} & 20 ans et moins & & & 2,19 & **** & & & 2,33 & **** \\
\hline & 25 ans et moins & & & ref. & & ref. & & ref. & \\
\hline & 30 ans et moins & & & 0,48 & **** & & & 0,73 & *** \\
\hline & 35 ans et moins & & & 0,29 & **** & & & 0,40 & ***** \\
\hline \multirow{7}{*}{ DIPLÔME } & Aucun diplôme & & & 2,76 & **** & & & 2,57 & ***** \\
\hline & Brevet & & & 1,86 & **⿻丷木 & & & 2,79 & **** \\
\hline & CAP, BEP ou équivalent & & & 1,24 & & & & 1,95 & **** \\
\hline & $\begin{array}{l}\text { Baccalauréat technique / } \\
\text { professionnel }\end{array}$ & & & 1,12 & & & & 1,39 & \\
\hline & Baccalauréat général & & & ref. & & & & ref. & \\
\hline & $\begin{array}{l}\text { Diplôme du supérieur court } \\
(\mathrm{bac}+2)\end{array}$ & & & 0,83 & & & & 0,77 & \\
\hline & Diplôme du supérieur long & & & 0,74 & & & & 0,80 & \\
\hline
\end{tabular}




\begin{tabular}{|c|c|c|c|c|c|}
\hline \multirow{4}{*}{$\begin{array}{l}\text { ORIGINE SOCIALE DES } \\
\text { DEUX PARENTS }\end{array}$} & $\begin{array}{l}\text { Agriculteurs, artisans et } \\
\text { commerçants }\end{array}$ & 1,14 & & 1,16 & \\
\hline & $\begin{array}{l}\text { Cadres et prof, sup, \& prof. } \\
\text { intermédiaires }\end{array}$ & 0,88 & & 0,92 & \\
\hline & $\begin{array}{l}\text { Ouvriers et employés } \\
\text { qualifiés }\end{array}$ & 0,76 & & 1,23 & \\
\hline & $\begin{array}{l}\text { Ouvriers et employés non } \\
\text { qualifiés }\end{array}$ & réf. & & réf. & \\
\hline \multirow{2}{*}{$\begin{array}{l}\text { RAPPORT À L'ACTIVITÉ } \\
\text { DE LA MĖRE }\end{array}$} & Mère active & Réf. & & Réf. & \\
\hline & Mère toujours inactive?? & 0,89 & & 1,08 & \\
\hline \multirow{2}{*}{ HABITAT } & $\begin{array}{l}\text { Hors zone urbaine défa- } \\
\text { vorisée }\end{array}$ & réf. & & réf. & \\
\hline & Zone urbaine défavorisée & ।,88 & ***** & 1,25 & \\
\hline \multirow{2}{*}{ RÉSIDENCE } & Hors unité urbaine Paris & réf. & & réf. & \\
\hline & Unité urbaine Paris & 0,83 & & 0,74 & * \\
\hline
\end{tabular}

Les seuils de significativité sont respectivement égaux à $1 \%$ (****), $5 \%$ (**) et $10 \%$ (*).

Source: enquête «Trajectoires et Origines», 2008, Ined-Insee

Champ: jeunes actifs âgés de 18 à 35 ans en 2008 ayant achevé leur formation initiale.

Lecture: Parmi les descendants d'immigrés nord-africains actifs, en comparaison de la population majoritaire masculine, la probabilité d'être chômeur plutôt qu'en emploi s'élève à 2,3 (OR), ce qui signifie que la probabilité d'être au chômage plutôt qu'occupé est 2,3 fois plus élevée chez les descendants d'immigrés nord-africains que dans la population majoritaire.

\subsection{LES FORMES D'EMPLOI}

\section{Une précarité sélective}

La période des trente glorieuses correspondant à l'avènement de la société salariale a vu s'instituer une norme d'emploi caractérisé par la stabilité et la durée de l'emploi que symbolise le contrat de travail à durée indéterminée et à temps plein. À partir de la seconde moitié des années 1970 et l'apparition d'un chômage structurel vont se multiplier des formes particulières d'emploi souvent qualifiées «d'atypiques», marquées par l'emploi à temps partiel ou à durée limitée qui précarisent les situations d’emploi et les salariés. Plusieurs catégories de population, tels que les jeunes ou les femmes, sont plus particu- 
lièrement assujetties à ces nouvelles formes d'emploi précaires : qu’en est-il des différentes catégories de descendants d’immigrés?

Les résultats montrent que même chez les jeunes, la norme d'emploi reste le contrat à durée indéterminée et à temps plein, y compris dans la population féminine qui est touchée par les différentes formes d’emploi temporaire, mais aussi par la diffusion du temps partiel (voir Tableau 4). Pour autant, le degré de précarisation diffère selon les catégories de descendants tant chez les hommes que chez les femmes.

Tableau 4: Types de contrat et temps de travail en 2008 selon l'origine et le sexe $[\mathrm{H} / \mathrm{F}]$ (en \%)

\begin{tabular}{|c|c|c|c|c|c|c|c|c|}
\hline & & \multicolumn{3}{|c|}{ EMPLOIS TEMPORAIRES } & \multirow[b]{2}{*}{$\begin{array}{c}\text { ENSEMBLE } \\
\text { DES EMPLOIS } \\
\text { TEMPORAIRES }\end{array}$} & \multicolumn{2}{|c|}{$\begin{array}{c}\text { EMPLOIS À DURÉE } \\
\text { INDÉTERMINÉE }\end{array}$} & \multirow[b]{2}{*}{$\begin{array}{l}\text { TEMPS } \\
\text { PARTIEL } \\
\text { (tous } \\
\text { contrats) }\end{array}$} \\
\hline & & $\begin{array}{c}\text { TRAVAIL } \\
\text { INTÉRIMAIRE }\end{array}$ & $\begin{array}{c}\text { FORMES } \\
\text { PARTICULIĖRES } \\
\text { D'EMPLOI } \\
\text { (hors CDD } \\
\text { et travail } \\
\text { intérimaire) } \\
\end{array}$ & $\begin{array}{c}\text { CONTRAT } \\
\text { À DURÉE } \\
\text { DÉTERMINÉEE } \\
\text { (CDD) }\end{array}$ & & $\begin{array}{c}\text { CDI A } \\
\text { TEMPS } \\
\text { COMPLET }\end{array}$ & $\begin{array}{l}\text { CDI A } \\
\text { TEMPS } \\
\text { PARTIEL }\end{array}$ & \\
\hline \multirow{2}{*}{$\begin{array}{l}\text { POPULATION } \\
\text { MAJORITAIRE }\end{array}$} & $\mathrm{H}$ & 5,7 & 1,5 & 10,1 & 17,3 & 79,5 & 3,1 & $22,0 *$ \\
\hline & $\mathrm{F}$ & 1,2 & 1,6 & 17,3 & 20,1 & 65 & 15 & 37,1 \\
\hline \multirow{2}{*}{$\begin{array}{l}\text { AFRIQUE DU } \\
\text { NORD }\end{array}$} & $\mathrm{H}$ & 9,5 & 3,2 & 14,2 & 26,9 & 70,3 & 2,9 & 36,4 \\
\hline & $\mathrm{F}$ & 4,2 & 3,5 & 18,5 & 26,2 & 63,7 & 10,2 & 42,2 \\
\hline \multirow{2}{*}{$\begin{array}{l}\text { AFRIQUE SUB- } \\
\text { SAHARIENNE }\end{array}$} & $\mathrm{H}$ & 6,5 & 3,7 & 16,3 & 26,5 & 70,7 & 2,9 & 39,5 \\
\hline & $\mathrm{F}$ & 2,7 & 5,3 & 25 & 33 & 60,9 & 6 & 39,2 \\
\hline \multirow{2}{*}{$\begin{array}{l}\text { ASIE DU } \\
\text { SUD-EST }\end{array}$} & $\mathrm{H}$ & 4,5 & 4,4 & 9,6 & 18,5 & 74,9 & 6,5 & 32,1 \\
\hline & $\mathrm{F}$ & 4,9 & 1,2 & 25,5 & 31,6 & 60,8 & 7,7 & 29,8 \\
\hline \multirow{2}{*}{ TURQUIE } & $\mathrm{H}$ & 8,1 & 0,7 & 10,7 & 19,5 & 78,4 & 2,1 & 42,7 \\
\hline & $\mathrm{F}$ & 3,4 & 1,8 & 27,7 & 32,9 & 58,4 & 8,8 & 50,5 \\
\hline \multirow{2}{*}{ PORTUGAL } & $\mathrm{H}$ & 5,2 & 3,2 & 7,1 & 15,5 & 79,5 & 5 & 17,5 \\
\hline & $\mathrm{F}$ & 3,4 & 1,8 & 27,7 & 32,9 & 58,4 & 8,8 & 24,2 \\
\hline \multirow{2}{*}{$\begin{array}{l}\text { EUROPE } \\
\text { (HORS } \\
\text { PORTUGAL) } \\
\end{array}$} & $\mathrm{H}$ & 4,4 & 0,4 & 11,4 & 16,2 & 78,8 & 5,1 & 19,3 \\
\hline & $\mathrm{F}$ & 3,1 & 0,6 & 11,4 & 15,1 & 73,6 & 11,4 & 33,9 \\
\hline \multirow{2}{*}{ AUTRES PAYS } & $\mathrm{H}$ & 6,6 & 3 & 14,4 & 24 & 73,1 & 2,9 & 19,8 \\
\hline & $\mathrm{F}$ & 0,8 & 2,1 & 16 & 18,9 & 64,3 & 16,8 & 29,3 \\
\hline
\end{tabular}

Source: enquête «Trajectoires et Origines», 2008, Ined-Insee.

Champ: jeunes actifs âgés de 18 à 35 ans en 2008 ayant achevé leur formation initiale.

Lecture: 5,7\% des actifs de la population majoritaire âgés de 18 à 35 ans sont intérimaires en 2008. *22\% des hommes de la population majoritaire travaillent à temps partiel en 2008 (soit 78\% à temps complet).

Parmi les femmes de même origine, $42 \%$ travaillent à temps partiel, $42,2 \%$ à temps complet. 
Dans la population masculine, l'emploi temporaire ou à temps partiel est surreprésenté parmi les actifs originaires d'Afrique du Nord et d'Afrique subsaharienne (plus d'un quart). On note aussi que le travail intérimaire, qui constitue une forme d'emploi temporaire plus instable que le contrat à durée déterminée (CDD), est également surreprésenté parmi les hommes descendants d'immigrés nord-africains et aussi de Turquie. Le temps partiel, qui s'apparente le plus souvent à du sous-emploi, n’épargne pas les hommes, en particulier les descendants des immigrations nord-africaines, d'Afrique subsaharienne, de Turquie ou du sud-est asiatique dont plus du tiers exercent à temps partiel contre $22 \%$ dans la population majoritaire (voir Tableau 4). En revanche, les formes d'emploi des descendants d'immigrés européens et du Portugal sont assez proches de la population majoritaire et ces jeunes travaillent moins souvent à temps partiel que les autres catégories de descendants, même si les CDI à temps partiel y sont sensiblement surreprésentés (Tableau 4). Au vu des conditions d'emploi, comme dans l'accès au premier emploi ou face au chômage, on retrouve la proximité entre la descendance européenne ou portugaise et la population majoritaire. Dans la population masculine, ces deux catégories de descendants exemplifient le modèle de l'intégration socio-économique. En revanche, la destinée des descendants d'immigrés nord-africains ou de l'immigration subsaharienne s'en écarte régulièrement et correspond mieux au modèle dit de l'assimilation segmentée.

Chez les femmes, l'emploi temporaire sous toutes ses formes concerne près du tiers des descendantes d'Afrique subsaharienne, d’Asie du Sud-Est ou de Turquie et un quart des descendantes du Maghreb. À cette précarité, s’ajoute la proportion élevée d'emploi à temps partiel (qu'il s'agisse de CDD ou de CDI) qui atteint 37 \% dans la population majoritaire (contre $22 \%$ chez les hommes de même origine), mais qui s'élève à $42 \%$ chez les descendantes de l'immigration nord-africaine et à $50 \%$ chez les descendantes d'immigrés turcs où le temps partiel est en passe de devenir la norme. Si dans l'ensemble les femmes travaillent plus souvent à temps partiel que les hommes, les écarts de sexe sous ce rapport sont plus ou moins marqués au sein des groupes. Ainsi, les différences entre hommes et femmes sont-elles faibles parmi les descendants d'Afrique subsaharienne et d'Asie du Sud-Est alors qu'elles sont plus prononcées dans les autres catégories. 
Afin de prendre la mesure de la précarisation sélective que subissent certaines catégories de descendants sur le marché du travail, nous avons pris en compte des facteurs susceptibles d’agir sur la qualité de l'emploi des salariés et plus particulièrement sur l'occupation d'un emploi sous contrat à durée indéterminée (CDI) et à temps plein, tels que le niveau de diplôme, l'âge, l'origine sociale, le secteur économique de l'emploi occupé, la zone de résidence. Les résultats de la modélisation - non présentée ici - montrent que les facteurs comme l'âge ou le secteur d’activité de l'emploi, mais aussi la région ou le quartier de résidence et bien évidemment le niveau de diplôme interagissent sur les chances d'occuper un emploi stable et durable tant dans la population masculine que féminine. Néanmoins, chez les hommes, les fils d'immigrés nord-africains et subsahariens apparaissent toujours pénalisés, toutes choses étant égales par ailleurs, en bénéficiant moins que les autres d'un contrat à durée indéterminée et à temps plein.

Toutes choses étant égales par ailleurs, les descendants d'origine turque lorsqu'ils sont salariés ne se distinguent pas de la population majoritaire à l'aune de la stabilité dans l'emploi, mais nous savons qu'ils affichent par ailleurs une plus forte propension à devenir indépendants.

Ces résultats confirment la sélectivité de la précarité de l'emploi et vont dans le sens de l'hypothèse d'une segmentation des trajectoires professionnelles et des conditions d'emploi des populations issues de l'immigration qui aboutit à une infériorisation socio-économique des descendants d'immigrés nord-africains et d’Afrique subsaharienne de sexe masculin. L’analyse séparée des sexes permet aussi de mettre au jour le caractère sexué et parfois contradictoire de la pénalisation des origines migratoires : les divisions entre origines migratoires ne font pas disparaître pour autant les rapports sociaux de sexe. 


\section{BIBLIOGRAPHIE}

BEAUCHEMIN C., HAMEL C., LESNE M., SIMON P., 20I0, «Les discriminations: une question de minorités visibles», Population et Sociétés, 466, INED, p.4.

BORREL C., LHOMMEAU B., 2010, «Être né en France d'un parent immigré », Insee Première, 1287, p. 4.

BOURDIEU P., 1974. «Avenir de classe et causalité du probable», Revue française de Sociologie, XV, 1974, p. 3-42.

BRINBAUM Y., WERQUIN P., 1997, «Enfants d'immigrés: un accès au travail difficile. Des itinéraires spécifiques», Informations Sociales, 62, p. 32-4I.

-, 1999, «Transition into the labour market of young people from immigrant families: differentiated pathways », in European Research Network on Transitions in Youth, D. Raffe, R. Van der Velden, P. Werquin (eds), «Education, the Labour Market and Transitions in Youth: Cross-National Perspectives», CES, Edinburgh, p. 147-164.

-, 2004, «Des parcours semés d'embûches: I'insertion professionnelle des jeunes d'origine maghrébine en France», in Marché du travail et genre, MaghrebEurope, L. Achy, J. de Henau, K. Kateb, J. Laufer, C. Marry, M. Maruani (éds.), Dulbea, Bruxelles, p. 145-166.

BRINBAUM Y., HAMEL C., PRIMON J-L, SAFI M., SIMON P., 20I0, «Discriminations», chapitre 17, Document de travail, I68, INED, p. I29-136.

BRINBAUM Y., MOGUEROU L., PRIMON J-L, 20I0. «Parcours et expériences scolaires des jeunes descendants d'immigrés en France », chapitre 6, Document de travail, 168, INED, p.47-53.

BRINBAUM Y., GUEGNARD C., 2012a, «Le sentiment de discrimination des descendants d'immigrés: reflet d'une orientation contrariée et d'un chômage persistant», Agora débats / jeunesses, 61, p. 7-20.

-, 2012b, «Parcours de formation et d'insertion des jeunes issus de l'immigration au prisme de l'orientation», Formation Emploi, |18, p. 61-82.

DE RUDDER V., POIRET C., VOURC'H F., 2000, L'Inégalité raciste. L'universalisme républicain à l'épreuve, Paris, PUF. 
DUPRAY A., MOULLET S., 2004, «Quelle discrimination à l'encontre des jeunes d'origine maghrébine à l'entrée du marché du travail en France?», in Marché du travail et genre, Maghreb-Europe, L. Achy, J. de Henau, K. Kateb, J. Laufer, C. Marry, M. Maruani (éds), Dulbea, Bruxelles, p. 167-182.

FRICKEY A., PRIMON J-L, 2002, «Jeunes issus de l'immigration: les diplômes de l'enseignement supérieur ne garantissent pas un égal accès au marché du travail», Formation Emploi, 79, p. 31-49.

-, 2006 «Une double pénalisation pour les non-diplômées du supérieur d’origine nord-africaine?», Formation Emploi, 94 , p. 27-43.

FRICKEY A., MURDOCH J., PRIMON J-L., 2004 «Les jeunes issus de l'immigration. De l'enseignement supérieur au marché du travail», Bref, 205, CEREQ, 4 p.

GAYMU J., PARANT A., 1996, «Les débuts dans la vie active des jeunes immigrés ou d'origine étrangère», Espaces Populations Sociétés, 2/3, p. 439-455.

LAACHER S., SAYAD A., 1998, «Insertion, intégration, immigration: la définition des mots est toujours un enjeu des luttes sociales», in Les Jeunes, l'insertion, l'emploi, B. Charlot, D. Glasman (éds), PUF, p. 166-185.

LHOMMEAU B., MEURS D., PRIMON J-L., 20I0, «Situation au marché du travail des 18-50 ans selon l'origine et le sexe». Document de travail, 168, Chapitre 7, INED, p. 55-62.

LORCERIE, F., 2000 «La lutte contre les discriminations ou l'intégration requalifiée», VEl enjeux, I21, p.69-81.

MEURS D., PAILHE A., SIMON P., 2005, «Immigrés et enfants d'immigrés sur le marché du travail: une affaire de génération?», in Histoires de familles, Histoires familiales. Les résultats de l'enquête famille de 1999, C. Lefèvre, A. Filhon (éds), Les Cahiers de l'INED, I56, INED, p.461-482.

PORTES A., ZHOU M., 1993, «The New Second Generation: Segmented Assimilation and Its Variants among post 1965 immigrant youth», The Annals of the American Academy of Political and Social Sciences (AAPSS), 530, p. 74-96.

PORTES A., FERNANDEZ-KELLY P., HALLER W., 2009, «The adaptation of the immigrant second generation in America: A theoretical overview and recent evidence », Journal of Ethnic and Migration Studies. 35-7. p. I077-1 04. 
RICHARD J-L., 1997, Dynamiques démographiques et socio-économiques de l'intégration des jeunes générations d'origine immigrée en France. Étude à caractère longitudinal (1975-1990). Thèse pour le doctorat ès Sciences Économiques, IEP-Paris, Juillet.

SAFI M., 2006 «Le processus d'intégration des immigrés en France: inégalités et segmentation », Revue française de sociologie, 47, p. 3-48.

SILBERMAN R., FOURNIER I., 1999, «Les enfants d'immigrés sur le marché du travail: les mécanismes d'une discrimination sélective», Formation Emploi, 65, p. 31-55.

-, 2006a, «Jeunes issus de l'immigration. Une pénalité à l'embauche qui perdure... », Bref, 226, CEREQ, 4 p.

-,. 2006b, «Les secondes générations sur le marché du travail en France: une pénalité ethnique ancrée dans le temps. Contribution à la théorie de l'assimilation segmentée», Revue Française de Sociologie, 47-2, p. 243-292.

SIMON P., 1998, «La discrimination: contexte institutionnel et perception par les immigrés», Hommes et migrations, «Le racisme à l'œuvre », 1211, p.49-67.

TRIBALAT M., 1995, Faire France: une grande enquête sur les immigrés et leurs enfants, Paris, La Découverte.

-, 1995, «Les immigrés et leurs enfants», Population et Sociétés, 300, 4 p.

TRIBALAT M., RIANDEY B., SIMON P., 1996, De l'immigration à l'assimilation. Enquête sur les populations d'origine étrangère en France, Paris, La Découverte.

ZHOU M., 1997, «Segmented assimilation: issues, controversies, and recent research on the new generation», International Migration Review, 31-4, p. 975-1008. 
